\title{
Impact of Time-to-Reperfusion on Outcome in Patients with Poor Collaterals
}

\author{
(D)Y.-H. Hwang, D.-H. Kang, Y.-W. Kim, Y.-S. Kim, S.-P. Park, and D.S. Liebeskind
}

it

\begin{abstract}
BACKGROUND AND PURPOSE: The relationship between reperfusion and clinical outcome is time-dependent, and the effect of reperfusion on outcome can vary on the basis of the extent of collateral flow. We aimed to identify the impact of time-to-reperfusion on outcome relative to baseline angiographic collateral grade in patients successfully treated with endovascular revascularization for acute large-vessel anterior circulation stroke.
\end{abstract}

MATERIALS AND METHODS: Two hundred seven patients were selected for analysis from our prospectively maintained registry. Inclusion criteria were M1 MCA \pm ICA occlusions, onset-to-puncture time within 8 hours, and successful endovascular reperfusion. Baseline angiographic collateral grades were independently evaluated and dichotomized into poor (0-1) versus good (2-4). Multivariable analyses were performed to identify the effect of collateral-flow adequacy on favorable outcome on the basis of onset-to-reperfusion time and puncture-to-reperfusion time.

RESULTS: In the poor collateral group, the odds of favorable outcome significantly dropped for patients with onset-to-reperfusion time of $>300$ minutes or puncture-to-reperfusion time of $>60$ minutes (onset-to-puncture time: $\leq 300,59 \%$ versus $>300,32 \% ;$ OR, $0.24 ; P=$ .011 ; puncture-to-reperfusion time: $\leq 60,73 \%$ versus $>60,32 \% ;$ OR, $0.21, P=.011)$, whereas the probability of favorable outcome in the good collateral group was not significantly influenced by onset-to-reperfusion time or puncture-to-reperfusion time. In the subgroup lesionvolume growth analysis by using DWI, the effect of puncture-to-reperfusion time of $>60$ minutes was significantly greater compared with the effect of puncture-to-reperfusion time of $<60$ minutes in the poor collateral group $\left(\beta=41.6 \mathrm{~cm}^{3}, P=.001\right)$.

CONCLUSIONS: Time-to-reperfusion including onset-to-reperfusion time and puncture-to-reperfusion time in patients with poor collaterals is an important limiting factor for favorable outcome in a time-dependent fashion. Future trials may benefit from a noninvasive imaging technique to detect poor collaterals along with a strategy for rapid reperfusion.

ABBREVIATIONS: $\mathrm{HI}=$ hemorrhagic infarction; $\mathrm{OPT}=$ onset-to-puncture time; $\mathrm{ORT}=$ onset-to-reperfusion time; $\mathrm{PH}=$ parenchymal hematoma; $\mathrm{PRT}=$ punctureto-reperfusion time

$\mathbf{T}$ he restoration of antegrade perfusion to the ischemic territory is the principal goal of current acute stroke treatments because it is associated with better clinical outcomes and reduced mortality. ${ }^{1,2}$

Received June 25, 2014; accepted after revision August 10.

From the Departments of Neurology (Y.-H.H., Y.-W.K., S.-P.P.), Neurosurgery (D.-H.K.), Radiology (D.-H.K., Y.-W.K., Y.-S.K.), and Cerebrovascular Center (Y.-H.H., D.-H.K., Y.-W.K., Y.-S.K.), Kyungpook National University Hospital, Daegu, Korea; School of Medicine (Y.-H.H., D.-H.K., Y.-W.K., Y.-S.K., S.-P.P.), Kyungpook National University, Daegu, Korea; and UCLA Stroke Center (D.S.L.), University of California, Los Angeles, Los Angeles, California.

Y.-H. Hwang and D.-H. Kang contributed equally to this work.

Please address correspondence to Yang-Ha Hwang, MD, PhD, Department of Neurology and Cerebrovascular Center, Kyungpook National University Hospital, 130, Dongduk-ro, Jung-gu, Daegu, 700-721, Republic of Korea; e-mail: yangha.hwang@ gmail.com; @yangha73

- Indicates open access to non-subscribers at www.ajnr.org

$\equiv$ Indicates article with supplemental on-line tables.

$\checkmark$ Indicates article with supplemental on-line photo.

http://dx.doi.org/10.3174/ajnr.A4151
Also, the effect of reperfusion on outcome is time-dependent, and onset-to-reperfusion time (ORT) has emerged as an important time metric to show the benefit of endovascular treatment. ${ }^{3,4}$

However, the effect of reperfusion on outcome can vary on the basis of the extent of collateral flow. ${ }^{5-11}$ Baseline collateral flow, which is an important determinant of clinical recovery, can mitigate potential injury to ischemic brain tissue. ${ }^{12}$ Before adequate reperfusion occurs, the penumbral area can continue to be salvageable depending on the robustness of collateral flow, and its impact on infarct growth and subsequent clinical outcome may be time-dependent, along with reperfusion status. It can be speculated that in patients with poor collaterals, the relationship between reperfusion and clinical outcome may be more dependent on time-to-reperfusion because the odds of potential brain injury may be higher until reperfusion occurs.

We hypothesized that differential clinical response to reperfusion can be driven by the quality of baseline collaterals in a time- 


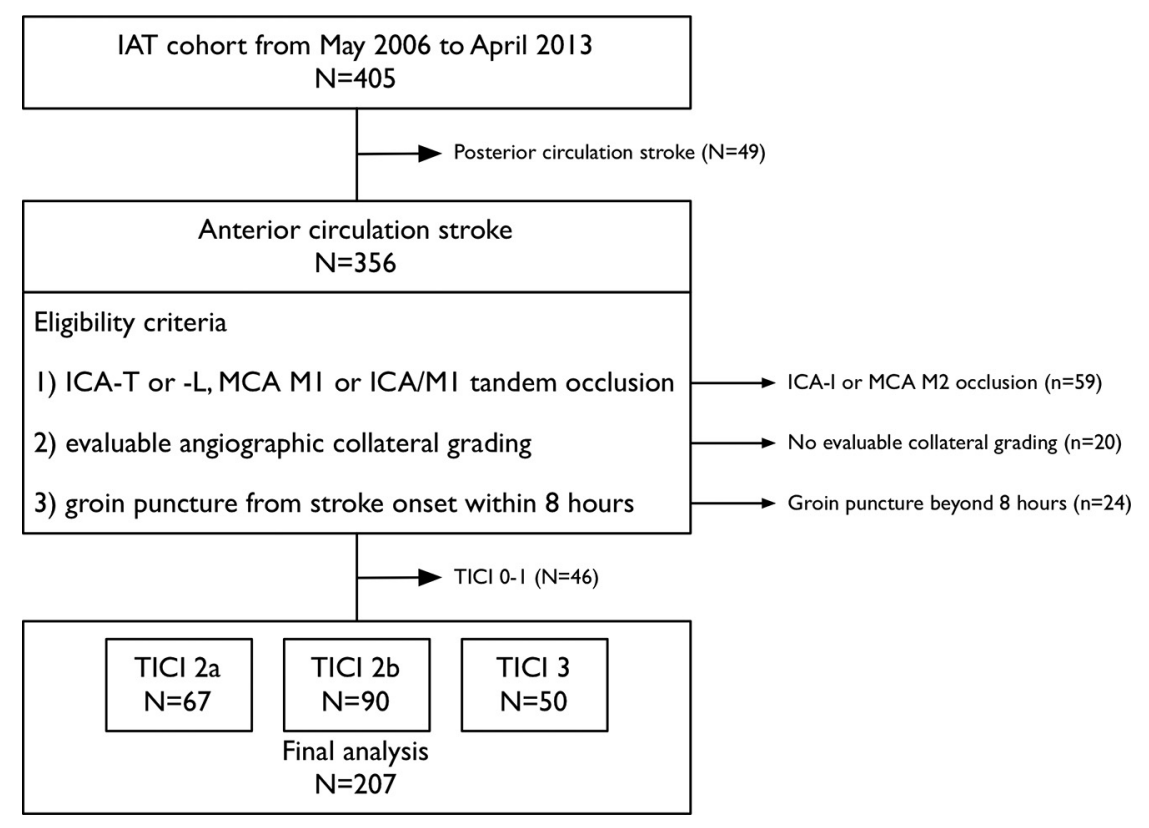

FIG 1. Flow chart description of patient selection and exclusion for the study. IAT indicates intra-arterial treatment.

dependent manner. We sought to determine the relationship among time-to-reperfusion, angiographic collateral-flow grade, and clinical and imaging outcome, which may show that there is a narrow therapeutic time window for patients having poor collaterals, despite angiographic reperfusion.

\section{MATERIALS AND METHODS}

Between May 2006 and April 2013, patients were retrospectively selected from a prospectively maintained acute stroke intra-arterial treatment registry at our institution. Eligibility for inclusion in this study was that patients met the following criteria: 1) They had an acute anterior circulation stroke with angiographically confirmed ICA-T or -L (-T or -L indicate the shape of occlusive lesion), MCA M1, or ICA/M1 tandem occlusion; 2) had evaluable angiographic imaging for collateral-flow grading; 3) had arterial puncture in the angiography suite room within 8 hours from time last-known well; and 4) achieved a sufficient angiographic reperfusion, which we defined as a TICI grades 2-3 (Fig 1). ${ }^{13}$ Within the 3-hour time window, intravenous rtPA was used, ${ }^{14,15}$ and patients showing a persistent ICA or MCA occlusion despite rtPA on TOF MRA or CTA were selected for endovascular treatment. We excluded patients who revealed well-developed parenchymal hypoattenuation on CT or hyperattenuation on FLAIR imaging, severe brain edema, intracerebral hemorrhage, or cardiopulmonary compromise. The local institutional review board approved this study for retrospective analysis.

Eligible patients underwent transfemoral cerebral angiography, including injection of both carotid arteries and the dominant vertebral artery through the late venous phase, under local or general anesthesia to define the angioarchitecture of the occluded vessel and to assess collateral flow from all possible sources. If the treatable ICA or MCA occlusion persisted, endovascular treatment was initiated. Treatment strategies were selected on the basis of available therapies at the time of angiography, which included intra-arterial thrombolytic infusion (urokinase or rtPA), mechanical clot disruption, mechanical thrombectomy including forced arterial suction thrombectomy or Solitaire thrombectomy (Covidien, Irvine, California), rescue intra-/extracranial stent, or a combination. ${ }^{16-18}$ Angiographic collateral-flow grade was evaluated with the American Society of Interventional and Therapeutic Neuroradiology/Society of Interventional Radiology collateral-flow grading system on pretreatment angiography. This angiographic scale assigns patients to grades $0-4$ according to the completeness and rapidity of collateral filling in a retrograde fashion. ${ }^{13}$ The angiographic collateral-flow grade was independently evaluated by an experienced neurologist (Y.-W.K.) and neuroradiologist (D.-H.K.) blinded to patient information and was dichotomized as poor (collateral-flow grades 0-1) and good (24). ${ }^{19}$ The $\kappa$ coefficient for interobserver agreement was 0.864 for each collateral grade. Disagreement was resolved by consensus. Reperfusion status was measured by the same raters and methods for the TICI scale. ${ }^{13}$

Information on demographic and clinical characteristics, medical history, admission blood pressure, and blood glucose levels was collected at baseline. The onset of stroke was defined as the time when the patient was last observed to be healthy. Stroke severity was assessed by using the NIHSS at baseline. The DWI at baseline was assessed by using ASPECTS. ${ }^{20}$ All patients underwent CT or MR imaging at 24-48 hours after the treatment. If there was evidence of hemorrhage, the subtype was classified as hemorrhagic infarction (HI), parenchymal hematoma (PH), $\mathrm{SAH}$, intraventricular hemorrhage, or mixed. ${ }^{21}$ Symptomatic intracranial hemorrhage was defined as any type of hemorrhage associated with an increase in the NIHSS score of $\geq 4$ within 24 hours. $^{22} \mathrm{~A}$ subset of 160 patients had undergone both pretreatment and posttreatment DWI (3-7 days from stroke onset). One experienced neurologist (Y.-W.K.) who was blinded to clinical status performed DWI lesion-volume calculation by using an open source image-analysis software (OsiriX Imaging Software; http://www.osirix-viewer.com). Functional status was assessed by using the mRS at 3 months, and favorable outcome was defined as an $\mathrm{mRS}$ of $\leq 2$ or equal to the prestroke $\mathrm{mRS}$ if the prestroke $\mathrm{mRS}$ was $>2{ }^{22,23}$

\section{Statistical Analysis}

Statistical analysis was performed by using the SPSS statistical package (Version 20.0; IBM, Armonk, New York). Bivariate comparisons were made by using the $\chi^{2}$ test or Fisher exact test as appropriate for categoric variables. The Student $t$ test was used for continuous variables, and the Mann-Whitney $U$ test was used for ordinal and continuous variables that had skewed distributions. Multivariable regression analysis was performed to identify the effect of collateral-flow grade on each outcome or lesion volume 
based on prespecified various time cutoff points. The results are presented as OR estimates of relative risk with a $95 \%$ CI or $\beta$ coefficient as appropriate. Probability values $<.05$ were statistically significant.

\section{RESULTS}

During the study period, 207 patients were included for analysis, and the details of exclusion are described in Fig 1. Overall, 76

\section{Baseline characteristics, imaging, and clinical outcomes} $(\mathrm{N}=207)^{\mathrm{a}}$

\begin{tabular}{|c|c|c|c|}
\hline \multirow[b]{2}{*}{ Characteristics } & \multicolumn{2}{|c|}{ Collateral-Flow Grade } & \multirow{2}{*}{$\begin{array}{c}P \\
\text { Value }\end{array}$} \\
\hline & $0-1(n=76)$ & $2-4(n=131)$ & \\
\hline Age (yr) & $69(62-76)$ & $67(58-73)$ & .105 \\
\hline Male & $50(65.8 \%)$ & $75(57.3 \%)$ & .226 \\
\hline Baseline NIHSS & $17(13-21)$ & $16(12-19)$ & .041 \\
\hline Baseline ASPECTS $_{\text {DWI }}{ }^{b}$ & $7(5-8)$ & $8(6-9)$ & $.004^{c}$ \\
\hline $\mathrm{SBP}(\mathrm{mm} \mathrm{Hg})$ & 148 (131-176) & $146(126-168)$ & .520 \\
\hline $\mathrm{DBP}(\mathrm{mm} \mathrm{Hg})$ & $82(72-90)$ & 82 (71-97) & .470 \\
\hline Blood glucose level (mg/dL) & $126(117-156)$ & $126(111-159)$ & .861 \\
\hline Occlusion site & & & .000 \\
\hline ICA-T or ICA-L & $38(50.0 \%)$ & $22(16.8 \%)$ & \\
\hline MCA M1 & $26(34.2 \%)$ & $103(78.6 \%)$ & \\
\hline ICA/M1 tandem & $12(15.8 \%)$ & $6(4.6 \%)$ & \\
\hline IV rtPA & $41(53.9 \%)$ & $62(47.3 \%)$ & .359 \\
\hline Onset-to-puncture (min) & $220(160-290)$ & $245(175-325)$ & .055 \\
\hline Puncture-to-reperfusion (min) & 77 (54-104) & $66(44-90)$ & .053 \\
\hline Onset-to-reperfusion (min) & $297(224-383)$ & $307(244-395)$ & .342 \\
\hline 3-Month favorable outcome & $35(46.1 \%)$ & $82(62.6 \%)$ & .021 \\
\hline Procedure-related complications & $4(5.3 \%)$ & $8(6.1 \%)$ & $1.000^{\circ}$ \\
\hline Symptomatic ICH & $4(5.3 \%)$ & $6(4.6 \%)$ & $1.000^{\circ}$ \\
\hline Ischemic brain edema & $7(9.2 \%)$ & $8(6.1 \%)$ & .406 \\
\hline Mortality & $11(14.5 \%)$ & $13(9.9 \%)$ & .324 \\
\hline
\end{tabular}

Note:- SBP indicates systolic blood pressure; DBP, diastolic blood pressure; $\mathrm{ICH}$, intracranial hemorrhage.

${ }^{a}$ Numbers in parentheses are median (interquartile range) or number (\%).

${ }^{\mathrm{b}}$ Two hundred one DWI images at baseline (72 in 0-1 and 129 in 2-4; 97.1\% in total) were available for ASPECTS , analysis.

${ }^{c}$ Fisher exact test for categoric variables/Mann-Whitney $U$ test for continuous variables.

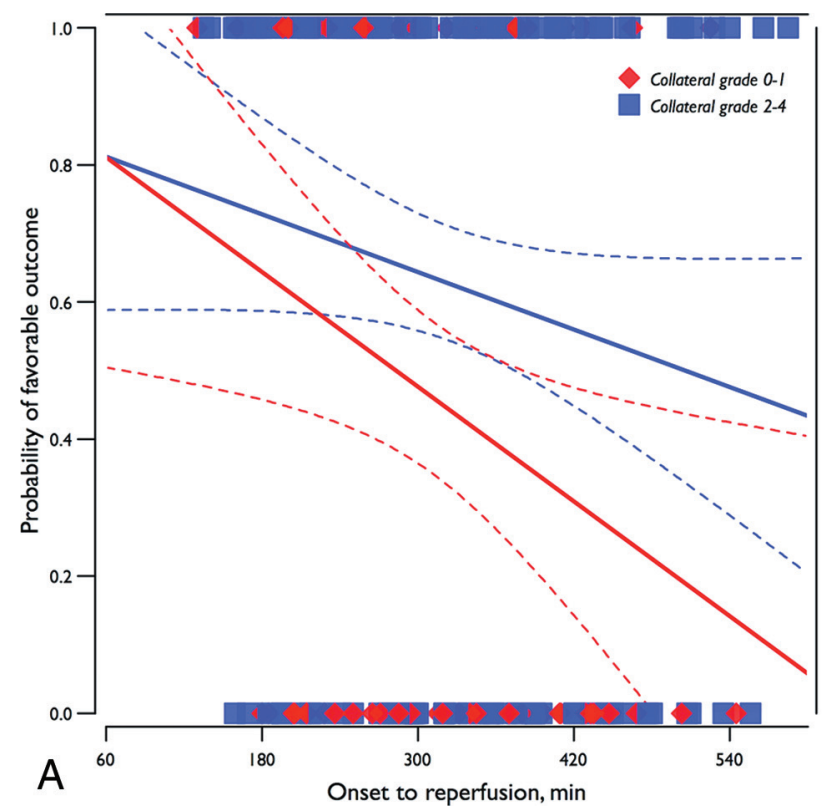

patients $(37 \%)$ were assigned to the poor collateral group (collateral-flow grades, $0-1)$, and 131 patients $(63 \%)$, to the good collateral group (collateral-flow grades, 2-4). The baseline characteristics and outcomes are described in the Table (On-line Table 1 in detail). Patients in the poor collateral group had a higher baseline NIHSS score, a lower baseline ASPECTS $_{\text {DWI }}$, a higher incidence of ICA-T or -L occlusion, and a lower rate of 3-month favorable outcome, all statistically significant.

\section{Time-to-Reperfusion and Favorable Outcome}

As shown in Fig $2 A$, the probability of favorable outcome decreased with every minute increase of ORT in the poor collateral group (OR, $0.99 ; 95 \% \mathrm{CI}, 0.99-1.00 ; P=.021$ ). The effect of ORT on the probability of favorable outcome in the good collateral group was also time-dependent, but it was not statistically significant (OR, 1.00; 95\% CI, 0.99-1.00; $P=.080)$. With 30-minute time intervals, the OR magnitude was 0.80 (95\% CI, 0.67-0.96; $P=.015)$ in the poor collateral group compared with the good collateral group (OR, 0.90; 95\% CI, 0.80-1.02; $P=.096$ ) after adjustment for age, baseline NIHSS score, and posttreatment TICI $2 \mathrm{~b}-3$ reperfusion. With a time cutoff point of 300 minutes for ORT, ORT of $>300$ minutes remained as an independent predictor of decreased odds for favorable outcome in patients with poor collateral flow (OR, $0.24 ; 95 \% \mathrm{CI}, 0.08-0.72 ; P=.011$ ) compared with good collateral flow (OR, $0.47 ; 95 \%$ CI, $0.21-1.08$; $P=.074)$ after adjustment for age, baseline NIHSS score, and posttreatment TICI $2 \mathrm{~b}-3$ reperfusion (Fig $3 A$ ).

We divided the ORT between onset-to-puncture time (OPT) and puncture-to-reperfusion time (PRT) to determine the effects of time spent on endovascular treatment. There was no timedependent effect on favorable outcome based on a time cutoff point of 240 minutes of OPT. Rather, as shown in Fig $2 B$, the probability of favorable outcome decreased with every minute

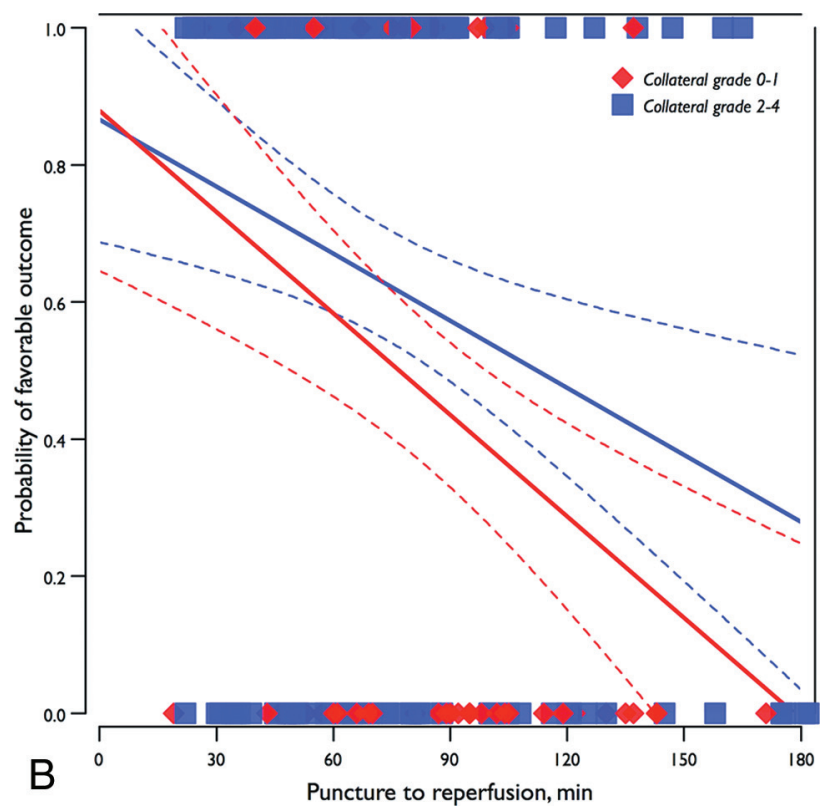

FIG 2. Unadjusted predicted probability of favorable outcome at 3 months by time-to-reperfusion based on collateral-flow grades $0-1$ (red line) versus 2-4 (blue line). Solid lines represent the probability of favorable outcome over onset-to-reperfusion time $(A)$ and puncture-to-reperfusion time $(B)$ as predicted by an unadjusted logistic regression model based on collateral-flow grade. Dashed lines show the $95 \%$ confidence intervals. Outcomes of each observed case are also indicated on the basis of collateral-flow grades 0-1 (red diamond) versus 2-4 (blue square). 
Effect of onset-to-reperfusion time on favorable outcome

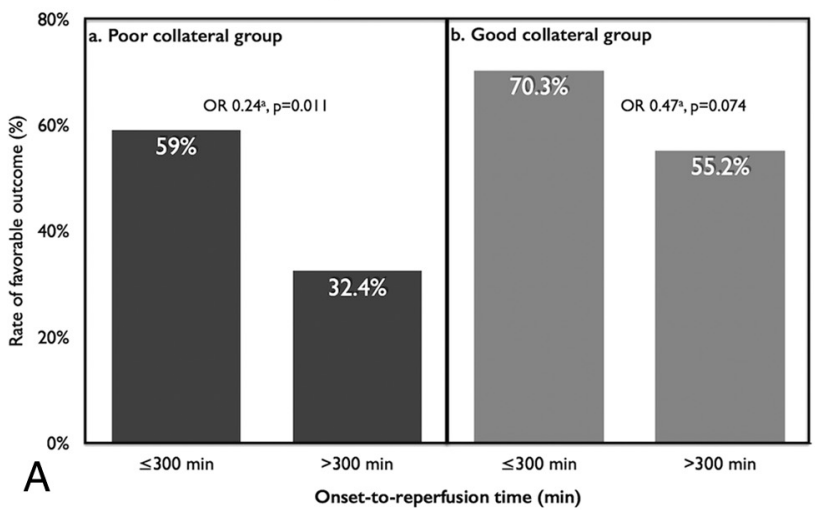

Effect of puncture-to-reperfusion time on hemorrhagic infarction

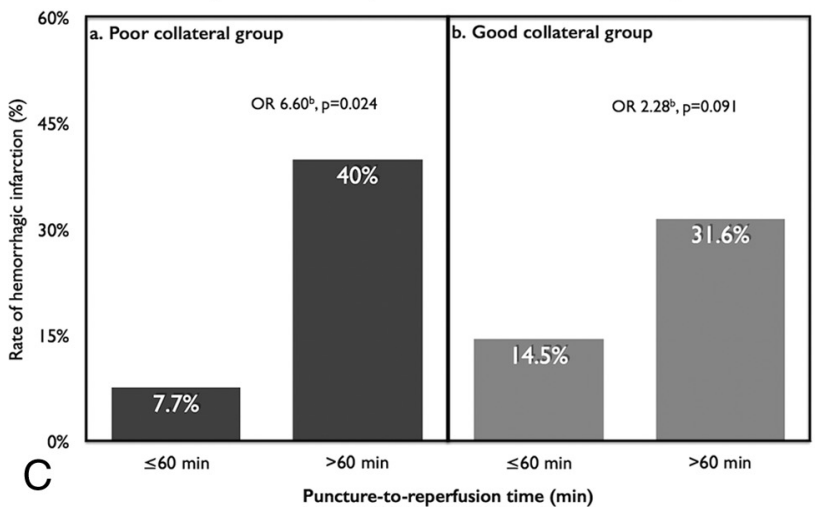

Effect of puncture-to-reperfusion time on favorable outcome

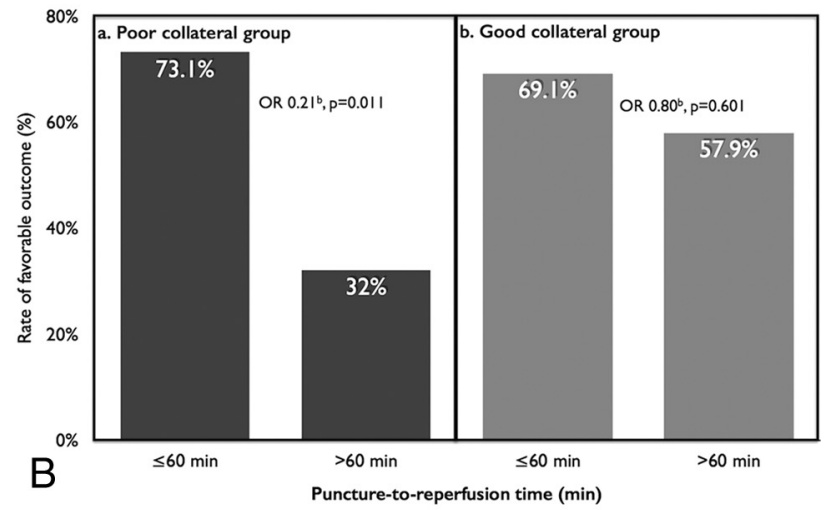

Effect of puncture-to-reperfusion time on lesion volume growth

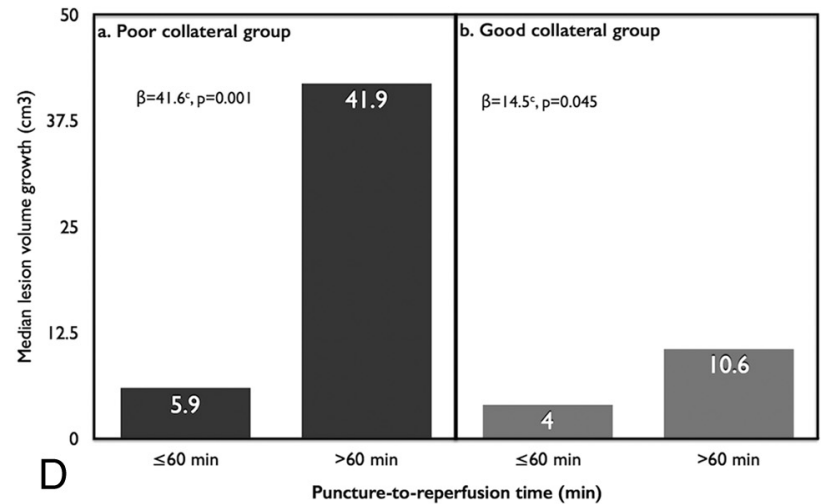

FIG 3. $A-D$, The effect of time-to-reperfusion on clinical and imaging outcomes based on collateral-flow grade. ${ }^{a}$ indicates logistic regression analysis adjusted for age, baseline NIHSS score, and posttreatment $\mathrm{TICI} 2 \mathrm{~b}-3$ reperfusion; ${ }^{b}$, logistic regression analysis adjusted for age, baseline NIHSS score, posttreatment $\mathrm{TICl} 2 \mathrm{~b}-3$ reperfusion, and onset-to-puncture time; ${ }^{c}$, linear regression analysis adjusted for age, baseline NIHSS score, posttreatment $\mathrm{TICl} 2 \mathrm{~b}-3$ reperfusion, and onset-to-puncture time.

increase of PRT in the poor collateral group (OR, 0.97; 95\% CI, $0.96-0.99 ; P=.001)$. The effect of PRT on the probability of favorable outcome in the good collateral group was also timedependent (OR, 0.99; 95\% CI, 0.98-1.00; $P=.005$ ). According to a time cutoff point of 60 minutes, PRT of $>60$ minutes remained an independent predictor of decreased odds of favorable outcome in patients with poor collateral flow (OR, 0.21; 95\% CI, $0.06-$ $0.70 ; P=.011)$ compared with good collateral flow (OR, 0.80; 95\% CI, 0.34-1.85; $P=.601$ ) after adjustment for age, baseline NIHSS score, posttreatment TICI $2 \mathrm{~b}-3$ reperfusion, and OPT (Fig $3 B$ ). The cutoff time points for ORT and PRT were chosen on the basis of sensitivity and specificity analyses, and details are described in the On-line Figure.

\section{Time-to-Reperfusion and Mortality/Intracerebral \\ Hemorrhage}

Unlike the significant relationship between the probability of favorable outcome and time-to-reperfusion, no statistically significant relationship was seen between the probability of mortality and prespecified time metrics based on collateral-flow grade. As to hemorrhage, the rate of HI was significantly increased in patients with ORT of $>300$ minutes in both the poor and good collateral groups. Likewise, a PRT of $>60$ minutes also increased the rate of $\mathrm{HI}$, which remained as an independent predictor of increased odds of $\mathrm{HI}(\mathrm{OR}, 6.60$; 95\% CI, 1.23-34.1; $P=.024)$ in the poor collateral group compared with the good collateral group (OR, 2.28; 95\% CI, 0.88-5.95; $P=.091$ ) after adjustment for age, baseline NIHSS score, posttreatment TICI $2 \mathrm{~b}-3$ reperfusion, and OPT (Fig 3C). The rate of PH was not significantly influenced by the prespecified time metrics based on collateralflow grade (On-line Table 2).

\section{Time to Reperfusion and DWI Lesion Volume}

A subset of 160 patients (of 207, 77.3\%) completed both pretreatment and posttreatment DWI (3-7 days from stroke onset). There was a significant imbalance regarding the completion of both DWIs between patients with a favorable outcome or those without; 108 of 117 (92.3\%) in patients with a favorable outcome versus 52 of $90(57.8 \%)$ in patients with an unfavorable outcome $(P=.000)$. At baseline, the median DWI lesion volume was 14.6 and $9.8 \mathrm{~cm}^{3}$ in the poor and good collateral group, respectively $(P=.115)$. The median DWI lesion volume at posttreatment and DWI lesion-volume growth were $56.6 \mathrm{~cm}^{3}$ and $31.1 \mathrm{~cm}^{3}$ versus $18.4 \mathrm{~cm}^{3}$ and $7.0 \mathrm{~cm}^{3}$ in the poor and good collateral groups, respectively $(P=.002$ and .001$)$. Multiple regression analysis was performed to elucidate the effect of prespecified time cutoff points on DWI lesion volume based on collateral-flow grade (Online Table 3). PRT of $>60$ minutes in the poor collateral group was an independent predictor of larger final lesion volume $(\beta=$ $\left.46.8 \mathrm{~cm}^{3} ; P=.004\right)$ and lesion-volume growth $\left(\beta=41.6 \mathrm{~cm}^{3} ; P=\right.$ $.001)$ after adjustment for age, baseline NIHSS score, posttreat- 


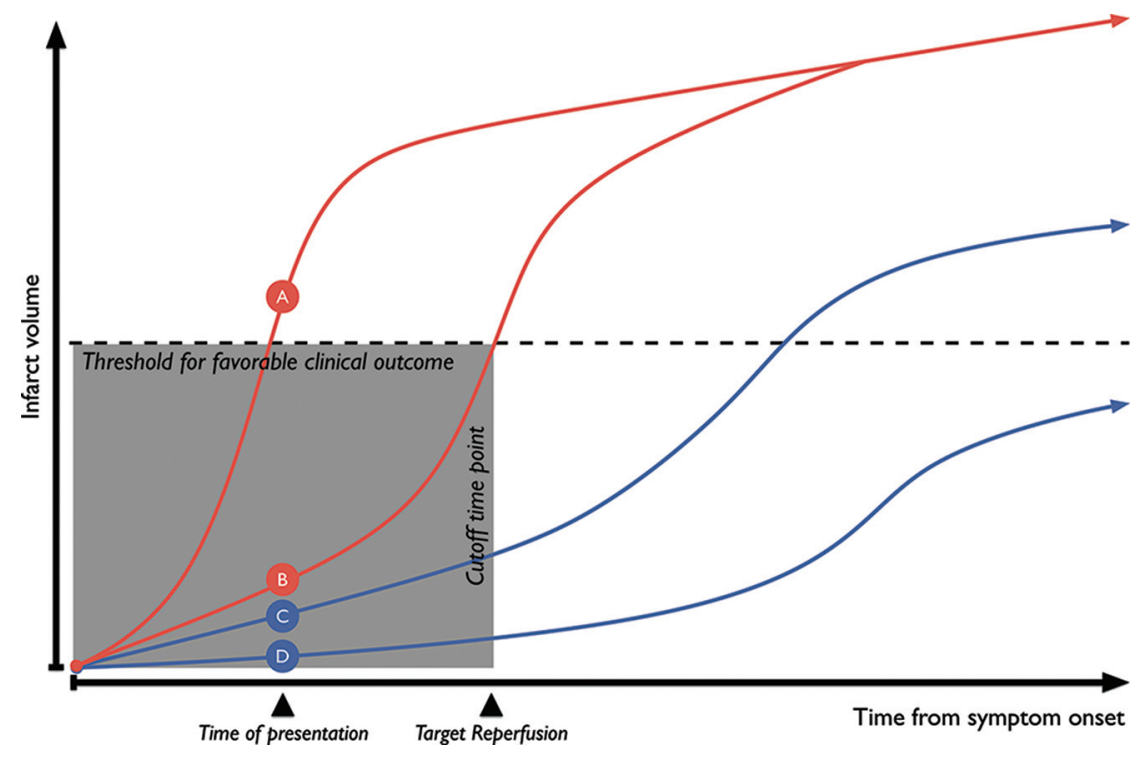

FIG 4. Simplified illustration, which represents the unique effect of time-to-reperfusion on clinical outcome based on proposed collateral scenarios (modified from Liebeskind's illustrations ${ }^{31}$ ). $A$, Universally poor collaterals, which may show large infarcts and severe neurologic deficits at the time of presentation. $B$, Rapidly failing collaterals, which may show small-to-medium infarcts and severe neurologic deficits at the time of presentation. $C$, Slowly failing collaterals, which may show small infarcts and moderate-to-severe neurologic deficits at the time of presentation. $D$, Universally good collaterals, which may show tiny-to-small infarcts and mild-to-moderate neurologic deficits at the time of presentation. In real clinical practice, patients in scenarios $B$ and $C$ can be ideal candidates for endovascular reperfusion. However, the clinical outcome is limited by time-to-reperfusion in scenario $B$ (eg, onset-to-reperfusion within 300 minutes or puncture-toreperfusion within 60 minutes in this study).

ment TICI $2 \mathrm{~b}-3$ reperfusion, and OPT (Fig 3D). In addition, a PRT of $>60$ minutes was associated with lesion-volume growth ( $\left.\beta=14.5 \mathrm{~cm}^{3}, P=.045\right)$ in the good collateral group, but the magnitude of the increment was not so large compared with the poor collateral group. The effect of final lesion volume and infarct volume growth remained significant from pretreatment DWI to reperfusion of $>150$ minutes in both groups; however, the magnitude of the increment was larger in the poor collateral group compared with the good collateral group.

\section{DISCUSSION}

In patients with acute ischemic stroke attributable to large-vessel occlusion, selecting a candidate who will benefit most from endovascular revascularization therapy to optimize the practice of endovascular treatment is important for improving clinical outcome. ${ }^{24}$ In consideration of that point, defining the optimal timeframe for reperfusion based on collateral-flow adequacy is crucial in averting further ischemic injury. ${ }^{25,26}$ Our study demonstrated that the evaluation of the collateral-flow status before the initiation of intra-arterial treatment could be a key marker to determine a timeframe for reperfusion with a probable favorable clinical outcome.

The effect of the quality of collateral flow on clinical outcome has been examined in previous reports, which show that good collateral flow is associated with favorable outcome. ${ }^{5-11}$ Also, the ORT in intra-arterial treatment was a main determinant factor in predicting favorable outcome. ${ }^{3,4}$ Regarding collateral flow and ORT as determinant factors of favorable outcome, reperfusion of $>300$ minutes from onset in a poor collateral group was associated with decreased odds of clinical improvement and favorable outcome from a small retrospective cohort. $^{27}$ Our study also showed that ORT is a critical determinant of favorable outcome in a time-dependent fashion (each minute, $30 \mathrm{~min}$ utes, or reperfusion of $>300$ minutes were all associated with a decreased chance of favorable outcome). Among the time variables affecting ORT, PRT was the main determinant of favorable outcome in the poor collateral group, which can be a more practical time metric to proceed or not proceed in the consideration of intra-arterial treatment. In the poor collateral group, PRT of $>60$ minutes was strongly associated with decreased odds of favorable outcome, which can be a practical marker for the determination of endovascular therapy termination. A previous study showed that the procedural time in intra-arterial treatment is a critical determinant of outcomes, ${ }^{28}$ and further negative impact on favorable outcome was shown in the poor collateral group in our study.

The extent of collateral flow has been associated with the rate of hemorrhage in patients with acute stroke undergoing intra-arterial treatment. ${ }^{19,29}$ In our study, the rate of hemorrhagic infarction was increased in the later time window (eg, ORT of $>300$ minutes and PRT of $>60$ minutes), especially in the poor collateral group; this change suggests that the ischemic area in the poor collateral group is more susceptible to reperfusion injury in a time-dependent manner. Unlike that previous study, ${ }^{29}$ we could not find any significant relationship between collateral-flow grade and parenchymal hematoma.

The status of collateral flow at the time of angiography has been shown to be associated with infarct growth in interaction with reperfusion. ${ }^{12}$ We tried to link the 3 -month favorable outcome and diffusion lesion volume to explain the inter-relationship between lesion-volume increments based on collateral-flow status. Perhaps because many cases of unfavorable outcome were excluded in this volumetric analysis, the relationship between ORT and lesion volume was not statistically significant on the basis of collateral-flow grade. However, we found that the chance of increased infarct volume is determined by the PRT and imaging-to-reperfusion time.

The main findings of this study were the following: 1) The probability of favorable outcome differed in a time-dependent manner on the basis of collateral-flow adequacy; 2) the rate of hemorrhagic infarction, which is a marker of reperfusion injury, increased with increasing ORT or PRT in the poor collateral group; and 3) lesionvolume enlargement was most prominent in patients with poor collaterals having a longer PRT or imaging-to-reperfusion time.

The results of this study should be interpreted with caution because it was not a randomized, controlled trial. Patients were treated with a variety of reperfusion therapies, including throm- 
bolytics and different types of endovascular therapy. Furthermore, although our patients were enrolled prospectively, the decision for endovascular treatment was based on our institutional treatment protocol and the attending physician's decision, so there was a chance of exclusion from our study if the patient had a sizable infarct volume with a large-vessel occlusion despite being within the treatable time window. Therefore, this study was limited by its retrospective nature and inherent case-selection bias. For example, for patients with poor collaterals at baseline, the chance of sizable infarct volume might be higher, and such a patient could be excluded from endovascular treatment. In addition, the exclusion of patients who failed reperfusion despite endovascular treatment might affect the results of our study because the chance of successful reperfusion could be higher in cases of good collaterals. ${ }^{11,30}$

\section{CONCLUSIONS}

In considering endovascular revascularization as a rescue treatment in patients with acute large-vessel occlusion, the status of baseline collateral flow at the time of angiography can be a crucial marker for favorable outcome and a limiting factor for lesion-volume increments in a time-dependent fashion (Fig 4). Therefore, limiting timeto-reperfusion, especially in patients with poor collaterals, is desirable in clinical practice, though our results should be confirmed in randomized trials or large datasets of prospective trials.

\section{ACKNOWLEDGMENTS}

The authors thank Wade Martin of Medical Research International for his critical English revision.

Disclosures: David S. Liebeskind_UNRELATED: Consultancy: consultant for the angiography core laboratory at the University of California, Los Angeles*; Grants/ Grants Pending: National Institutes of Health-National Institute of Neurological Disorders and Stroke.* *Money paid to the institution.

\section{REFERENCES}

1. Zaidat OO, Suarez JI, Sunshine JL, et al. Thrombolytic therapy of acute ischemic stroke: correlation of angiographic recanalization with clinical outcome. AJNR Am J Neuroradiol 2005;26:880-84

2. Rha JH, Saver JL. The impact of recanalization on ischemic stroke outcome: a meta-analysis. Stroke 2007;38:967-73

3. Khatri P, Abruzzo T, Yeatts SD, et al. Good clinical outcome after ischemic stroke with successful revascularization is time-dependent. Neurology 2009;73:1066-72

4. Mazighi M, Chaudhry SA, Ribó M, et al. Impact of onset-to-reperfusion time on stroke mortality: a collaborative pooled analysis. Circulation 2013;127:1980-85

5. Kucinski T, Koch C, Eckert B, et al. Collateral circulation is an independent radiological predictor of outcome after thrombolysis in acute ischaemic stroke. Neuroradiology 2003;45:11-18

6. Christoforidis GA, Mohammad Y, Kehagias D, et al. Angiographic assessment of pial collaterals as a prognostic indicator following intra-arterial thrombolysis for acute ischemic stroke. AJNR Am J Neuroradiol 2005;26:1789-97

7. Miteff F, Levi CR, Bateman GA, et al. The independent predictive utility of computed tomography angiographic collateral status in acute ischaemic stroke. Brain 2009;132:2231-38

8. Galimanis A, Jung S, Mono ML, et al. Endovascular therapy of 623 patients with anterior circulation stroke. Stroke 2012;43:1052-57

9. Souza LCS, Yoo AJ, Chaudhry ZA, et al. Malignant CTA collateral profile is highly specific for large admission DWI infarct core and poor outcome in acute stroke. AJNR Am J Neuroradiol 2012;33:1331-36

10. Liebeskind DS, Tomsick TA, Foster LD, et al. Collaterals at angiog- raphy and outcomes in the Interventional Management of Stroke (IMS) III trial. Stroke 2014;45:759-64

11. Liebeskind DS, Jahan R, Nogueira RG, et al. Impact of collaterals on successful revascularization in Solitaire FR with the intention for thrombectomy. Stroke 2014;45:2036-40

12. Bang OY, Saver JL, Buck BH, et al. Impact of collateral flow on tissue fate in acute ischaemic stroke. J Neurol Neurosurg Psychiatry 2008;79:625-29

13. Higashida RT, Furlan AJ, Roberts $\mathrm{H}$, et al. Trial design and reporting standards for intra-arterial cerebral thrombolysis for acute ischemic stroke. Stroke 2003;34:e109-37

14. National Institute of Neurological Disorders and Stroke rt-PA Stroke Study Group. Tissue plasminogen activator for acute ischemic stroke. N Engl J Med 1995;333:1581-87

15. Yamaguchi T, Mori E, Minematsu K, et al. Alteplase at $0.6 \mathrm{mg} / \mathrm{kg}$ for acute ischemic stroke within 3 hours of onset: Japan Alteplase Clinical Trial (J-ACT). Stroke 2006;37:1810-15

16. Hwang YH, Kang DH, Kim YW, et al. Outcome of forced-suction thrombectomy in acute intracranial internal carotid occlusion. J Neurointerv Surg 2013; (5 suppl 1):i81-84

17. Kang DH, Hwang YH, Kim YS, et al. Direct thrombus retrieval using the reperfusion catheter of the Penumbra system: forced-suction thrombectomy in acute ischemic stroke. AJNR Am J Neuroradiol 2011;32:283-87

18. Kang DH, Kim YW, Hwang YH, et al. Switching strategy for mechanical thrombectomy of acute large vessel occlusion in the anterior circulation. Stroke 2013;44:3577-79

19. Bang OY, Saver JL, Kim SJ, et al. Collateral flow averts hemorrhagic transformation after endovascular therapy for acute ischemic stroke. Stroke 2011;42:2235-39

20. Barber PA, Demchuk AM, Zhang J, et al. Validity and reliability of a quantitative computed tomography score in predicting outcome of hyperacute stroke before thrombolytic therapy: ASPECTS Study Group-Alberta Stroke Programme Early CT Score. Lancet 2000;355: 1670-74

21. Hacke W, Kaste M, Fieschi C, et al. Randomised double-blind placebocontrolled trial of thrombolytic therapy with intravenous alteplase in acute ischaemic stroke (ECASS II). Lancet 1998;352:1245-51

22. Saver JL, Jahan R, Levy EI, et al. Solitaire flow restoration device versus the Merci retriever in patients with acute ischaemic stroke (SWIFT): a randomised, parallel-group, non-inferiority trial. Lancet 2012;380:1241-49

23. van Swieten JC, Koudstaal PJ, Visser MC, et al. Interobserver agreement for the assessment of handicap in stroke patients. Stroke 1988;19:604-07

24. Lazzaro MA, Novakovic RL, Alexandrov AV, et al. Developing practice recommendations for endovascular revascularization for acute ischemic stroke. Neurology 2012;79:S243-55

25. Liebeskind DS. Collateral circulation. Stroke 2003;34:2279-84

26. Liebeskind DS, Sanossian N. How well do blood flow imaging and collaterals on angiography predict brain at risk? Neurology 2012; 79:S105-09

27. Ribo M, Flores A, Rubiera M, et al. Extending the time window for endovascular procedures according to collateral pial circulation. Stroke 2011;42:3465-69

28. Hassan AE, Chaudhry SA, Miley JT, et al. Microcatheter to recanalization (procedure time) predicts outcomes in endovascular treatment in patients with acute ischemic stroke: when do we stop? AJNR Am J Neuroradiol 2013;34:354-59

29. Christoforidis GA, Karakasis C, Mohammad Y, et al. Predictors of hemorrhage following intra-arterial thrombolysis for acute ischemic stroke: the role of pial collateral formation. AJNR Am J Neuroradiol 2009;30:165-70

30. Bang OY, Saver JL, Kim SJ, et al. Collateral flow predicts response to endovascular therapy for acute ischemic stroke. Stroke 2011; 42:693-99

31. Liebeskind DS. Imaging the future of stroke: I. Ischemia. Ann Neurol 2009;66:574-90 